Neurosurg Focus 13 (6):Article 5, 2002, Click here to return to Table of Contents

\title{
The use of electrical stimulation to enhance spinal fusion
}

\author{
Michael A. Morone, M.D., Ph.D., And Henry Feuer, M.D. \\ Deaconess Billings Clinic, Billings, Montana, and Indianapolis Neurosurgical Group, Indianapolis, \\ Indiana
}

\begin{abstract}
The goal of spinal arthrodesis is a solid osseous union across one or more spinal segments. A solid bone union after arthrodesis is commonly known as a spinal fusion. Surgeons have begun to understand the biomechanical and biological factors that influence the bone-healing process. One of the most commonly used adjuncts is spinal instrumentation. Instrumentation has increased the spinal fusion rate; however, fusion failure (that is, nonunion or pseudarthrosis) remains significant. A less commonly used adjunct is electrical stimulation (ES). Investigators in experimental studies have demonstrated the beneficial effects of ES on increasing the fusion rate. In this review the authors discuss the evidence concerning the benefits of ES as an adjunct to spinal arthrodesis. In addition, the different types of ES devices are described along with the current experimental and clinical evidence for each type of device.
\end{abstract}

KEY WORDS - spinal fusion - electrical stimulation • bone healing

The goal of a spinal fusion procedure is to produce a solid osseous union. Often this goal is not obtained and the resulting failure to fuse is known as a nonunion or pseudarthrosis. Many factors increase the rate of nonunion including prior surgery, multilevel arthrodesis, poor nutritional status, nicotine use, and certain medications such as steroid agents and nonsteroidal antiinflammatory drugs. Many of these factors can be modified to increase the rate of fusion. Nonetheless the nonunion rate can be a high as 35 to $40 \%$. One modality spine surgeons have used to enhance the fusion rates is ES.

Although the exact mechanism of electricity's beneficial effects on bone healing is not completely understood, ES has been performed for nearly 30 years to enhance spinal fusion. ${ }^{18}$ The clinical efficacy of ES on bone union has been more definitively demonstrated in long bones. Because of its efficacy in long bone healing, ES has been extrapolated for use in spinal arthrodesis procedures. In this article we review the effects of electricity on bone healing, the proposed mechanisms of ES on bone growth, describe the types of electrical stimulators, and review the studies examining the effectiveness of ES after spinal arthrodesis.

Abbreviations used in this paper: $\mathrm{BMD}=$ bone mineral desnsity; $\mathrm{BMP}=$ bone morphogenetic protein; $\mathrm{CMF}=$ combined magnetic field; DCES = direct current electrical stimulation; ES = electrical stimulation; PEMF = pulsed electromagnetic field; TGF $\beta=$ transforming growth factor- $\beta$.

\section{EFFECTS OF ELECTRICITY ON BONE}

It is known that under mechanical stress, electrical potentials are generated in bone., ${ }^{9,46}$ These piezoelectric effects are found to occur when enough shearing force is applied to the collagen fibers to induce them to slip past each other. ${ }^{27}$ Strain-related potentials due to mechanical deformation of bone are streaming potentials derived from the flow of a conductive fluid past a charged solid surface. The magnitude of bioelectrical potentials relates to the extent of remodeling or repair of bone. Whereas resting bone potentials range from 0.1 to $10 \mathrm{mV}$, ordinary physical activity produces electrical potentials of $20 \mathrm{mV}$. In areas of bone repair following fracture, potential gradients rise to 10 to $50 \mathrm{mV} / \mathrm{cm}$, with current densities of 5 to $15 \mu \mathrm{A} /$ $\mathrm{cm}^{2} .{ }^{1} \mathrm{~A}$ net negative charge along the bone surface is noted at bone-bone interfaces under compression. An example of this in the spine is an interbody fusion in which the graft is placed under tension so that it is compressed between the vertebral endplates. In areas of compressive strain, bone develops negative potentials of 10 to $100 \mathrm{mV}$ relative to areas of tension and responds with osteogenesis. ${ }^{1}$ Bone placed under tension-for example, a poorly loaded anterior spinal column graft or possibly a posterolateral intertransverse process graft-causes a net overall positive charge on the bone surface. With tension on a graft, the reverse of osteogenesis is more likely to occurthat is, bone resorption. Other investigators have experimentally demonstrated that ES and electromagnetic stimulation of bone augments angiogenesis, dampens os- 
teoclastic resorption, and promotes osteogenesis. ${ }^{8}$ Friedenberg and Brighton ${ }^{22}$ demonstrated the presence of another type of electrical potential that could be observed only in living bone and known as the bioelectrical or steady-state potential. This potential was noted to be electronegative in areas of bone undergoing active growth or repair compared with areas of resting bone.

Experimentally, the beneficial effects of electricity on bone healing have been demonstrated in long bone fracture models. In 1957, Fukuda and Yasuda ${ }^{23}$ showed that a continuous current of $1 \mu \mathrm{A}$ over 3 weeks produced new bone growth in rabbit femora. Electrical stimulation-induced osteogenesis had no relation to the piezoelectric mechanism other than polarity separation. Direct current delivered via electrodes in long bones results in osteogenesis around the negative electrode and resorption around the positive electrode. In 1974, Basset, et al., ${ }^{6}$ recorded increased osteogenesis in acute long bone fractures when exposed to coupled electromagnetic fields, a noninvasive technique.

Realizing the effects of ES on long bone healing, it is reasonable to consider the addition of electrical current to enhance the fusion rate after spinal arthrodesis. Clinically, the earliest report on the use of ES to improve the postoperative fusion rate was published nearly 30 years ago. The authors found that adjunctive ES improved the fusion rate in a diagnostically diverse group of patients undergoing both anterior and posterior spinal arthrodesis. ${ }^{18}$ In the next decade, only two brief reports appeared in which the effects of ES on spinal fusion were examined. Both of these reports were delivered as meeting presentations, (Brooks MD, Macys JR, unpublished data). In each, increased rates of fusion after posterior lumbar arthrodesis were associated with the use of implantable direct current electrical stimulators. More than 10 years would pass until a report on the use of fusion-related ES would be published in a peer-reviewed journal..$^{43}$ Although these the authors reported the benefits of ES on spinal fusion, different types of ES were used. To understand ES, a discussion concerning the various types of devices is needed.

\section{TYPES OF ELECTRICAL STIMULATION}

Electrical fields can be applied exogenously through three methods: 1) DECS, in which electrical current is surgically applied through implantation of a cathode into sites of bone repair and an anode in the nearby tissue; 2) PEMF, in which electrical fields are induced in bone through a PEMF that results in time-varying magnetic fields of 0.1 to $20 \mathrm{G}$ and voltage gradients of 1 to $10 \mu \mathrm{V}$ in an inductive coil; and 3) capacitive coupling, in which electrical fields can be introduced noninvasively by applying skin electrodes on sides contralateral to the bone to be stimulated. External potentials of 1 to $10 \mathrm{~V}$ provide potentials in tissue of 1 to $10 \mu \mathrm{V} / \mathrm{cm}$. Direct current ES and PEMFs are the two modalities most studied for the effects on spinal fusion. In addition, the authors of clinical studies have only reported ES use in lumbar arthrodesis. Their effects on thoracic and cervical regions have not yet been reported.

\section{Direct Current Electrical Stimulation}

In DCES an implantable device is used; it which con- sists of a hermetically sealed generator delivering a constant current of 20 to $40 \mu \mathrm{A}$ to the fusion site (depending on the model) through which two titanium cathodes are connected by insulated wires. This device typically remains functional for a minimum of 6 to 9 months postimplantation. At the discretion of the surgeon, the generator is often removed at 1 year postoperatively. During a typical posterolateral procedure, after decortication and just prior to placement of the bone graft material, the cathodes are placed in the lateral gutters touching the transverse processes to contact with as much viable bone as possible. Bone graft is then placed over the transverse processes. Care must be taken to use the graft to span and cover completely the area of the fusion, shielding the electrodes from any implanted internal fixation devices. This is done to prevent contact between the electrodes and the metallic implants, which could disable the electrical stimulator. Before closure, the generator is placed beneath the dorsal fascia along the paramedian region cephalad to the fusion area, or in the soft tissue proximal to the iliac crest. The generator should be placed in a comfortable tissue pocket so that raising of the skin contour is avoided or minimized. It is important to ensure that the generator (which functions as the anode) is in soft tissue and positioned 8 to 10 $\mathrm{cm}$ from the cathodes. The effective area of stimulation surrounding the cathodes is 5 to $8 \mathrm{~mm}$, and different geographical shapes allow for maximum contact with the graft material. ${ }^{3,15}$ The cathodes are powered by an implantable battery, which delivers a constant direct current for 6 to 9 months.

Possible Mechanisms of Action of DCES on Spinal Fusions. Although the exact mechanism of DCES on osteogenesis is not completely understood, a number of electrophysiological changes are known to occur during bone healing. It is thought that DCES enhances some of the following actions: the attraction of charged proteins and growth factors (electrophoresis); the movement of bone, cartilage, and endothelial cells to the fusion site (galvanotaxis); and polarization of cell membranes. Unique to DCES are specific chemical reactions known as faradic reactions at the cathode-bone interface. At the surface of the cathode the chemical reaction that occurs is as follows: $2 \mathrm{H}_{2} \mathrm{O}+4 \mathrm{e}^{-}+\mathrm{O}_{2}=4 \mathrm{OH}^{-}$. Additionally formation of $\mathrm{H}_{2} \mathrm{O}_{2}$ may occur. The formation of $\mathrm{OH}^{-}$and $\mathrm{H}_{2} \mathrm{O}_{2}$ at the surface of the cathode reduces the $\mathrm{PO}_{2}$ and slightly increases the $\mathrm{pH}$. Constant direct current is more effective in stimulating osteogenesis than pulsed direct current. ${ }^{13}$ Reduced $\mathrm{PO}_{2}$ has been noted experimentally in fracture calluses and in newly formed bone, because both growth plate cartilage and bone cells use a predominately anaerobic pathway. The aforementioned electrical field effects and the faradic products act together and separately to stimulate Ca uptake.,14-16 Alkalosis, a localized increase in $\mathrm{pH}$, stimulates osteoblast bone formation and mineralization whereas it inhibits bone resorption by osteoclasts. The result is that the rate of new bone formation exceeds bone resorption with the resultant net increase in bone growth. ${ }^{3}$

\section{Pulsed Electromagnetic Fields}

In contrast to the DCES, PEMF devices are not implanted but are externally worn as one or two coils that 
generate an electromagnetic field (a time-varying magnetic field and induced electrical field) across the area of the targeted fusion area. These coils may be incorporated into the brace and are usually worn 3 to 8 hours per day for 3 to 6 months after surgery. ${ }^{38}$ Whereas postoperative immobilization and rest lead to bone resorption of long bones, this osteoporosis can be reversed by applying PEMF stimulation. Improved angiogenesis, early bone union, and reduced bone resorption have been reported benefits of PEMF. ${ }^{5,8}$

The mechanism of action of PEMF stimulation on bone healing is less well understood than DCES. It is hypothesized that only the effects of the induced electrical field, and not the magnetic field, exert a biological action. Several hypotheses have been proposed. Some authors have maintained that there are PEMF-induced alterations in cell membrane potentials, whereas others have proposed that there are alteration of the molecular configuration of parathormone receptors, and still others have hypothesized that changes result in an increase in Ca influx into cells. ${ }^{17,19,20}$ Bassett alone $^{4}$ and with coworkers ${ }^{7}$ has shown that there is an increase in the calcification of bone repair initiated fibrocartilage that may further set the stage for vascularization. Aaron, et al., ${ }^{2}$ have demonstrated calcification increased in a rat model where demineralized bone powder was placed in the subcutaneous tissue of the abdominal flank. This model mimics the bone healing process precisely, researchers have found that the synthesis of cartilage molecules and the subsequent endochondral calcification were stimulated by PEMF. ${ }^{47}$

Investigations into the molecular biology of ES suggest that the electrical field may exert a modulation effect on the proliferation and differentiation of target cells and also stimulate matrix and growth factor production. Pulsed electromagnetic field has been shown to increased levels of BMP-2 and BMP-4 messenger RNA in rat calvarial osteoblasts when compared with controls. ${ }^{10}$ This effect was directly related to the duration of PEMF exposure and suggests that clinically applied PEMFs have a reproducible osteogenic effect in vitro, simultaneously inducing BMP-2 and -4 messenger RNA transcription. An increase in TGF $\beta$ was shown in atrophic and hypertrophic nonunion cells exposed to PEMF. Additionally in an experiment with M63 cells, PEMF demonstrated enhanced osteogenic differentiation in response to TGF $\beta .^{35}$

More recently, CMFs and capacitive coupling (see proceeding section) have been approved for use as an adjunct in spinal fusion. Much like the PEMF apparatus, these devices are worn externally and used for up to 9 months postoperatively. The $\mathrm{CMF}$ device differs from that of PEMF by superimposing the time-varying magnetic field onto an additional static magnetic field. The device is typically worn for 30 minutes daily. The rationale for the combined field with 30-minutes/day treatment was based data obtained in animal studies, in which investigators demonstrated increased bone stiffness at the 30-minute dose. The treatment effect, however, was far greater in this animal model with 24-hour/day treatment, indicating a dose response. ${ }^{48}$ In addition, a comparison of PEMF with CMF in a rabbit tibial osteotomy model showed the two signals to be very similar at equal treatment times, (Nepola JV, et al., unpublished data)

\section{Capacitive Coupling}

The capacitive coupling device uses small electrodes attached to the surface of the skin over the fusion area for constant 24-hour/day treatment; the batteries are changed daily and electrodes replaced periodically. The true mechanism of action, much like the PEMF and DCES, is not completely understood. Lorich, et al., ${ }^{36}$ however, have demonstrated the biochemical pathway by which the osteogenic response is elicited. Their study design included MC3T3-E1 and rat calvarial bone cells subjected to a capacitively coupled electric field of $20 \mathrm{mV} / \mathrm{cm}$. Chemical processing of the cells revealed the DNA content, which determined cell proliferation. A process of elimination and detection was postulated in which the biochemical path with known biochemical blocking agents that included verapamil, a Ca channel blocker, W-7, a calmodulin antagonist, indocin, a prostaglandin synthesis inhibitor, bromophenacyl bromide, a phospholipase A2 inhibitor, and neomycin, an inhibitor of the inositol phosphate cascade. Based on observations of cellular proliferation in electrically stimulated and control samples in the presence or absence of various combinations of these agents, it was hypothesized that the signal transduction pathway mediating the proliferative response of the test cells to electric field involved transmembrane $\mathrm{Ca}$ translocation or movement through voltage-gated $\mathrm{Ca}$ channels with a subsequent rise in levels of prostaglandin E2 and activation of calmodulin. It was also noted that the inosital phosphate pathway, dominant in mechanically stimulated bone cells, does not play a role in the proliferative response of bone cells to ES. ${ }^{36}$ Evidence also exists that a change in TGF $\beta_{1}$ messenger RNA occurs in bone cells in response to capacitive coupling. ${ }^{48}$

\section{EVALUATION OF ELECTRICAL STIMULATION DEVICES FOR USE IN SPINAL SURGERY}

Before assessing the scientific and clinical efficacy of these spinal fusion adjuncts, it must be noted that not all ES devices work in the same manner. Additionally bone fusions do not physiologically or biomechanically occur in the same manner. The physiological and biomechanical forces acting upon the healing of anterior interbody and posterolateral fusions are quite different. The electrical effects of bone placed in compression results in the formation of electrically negative charges on the bone surface. As has been mentioned, negative charges induce osteogenesis. In posterolateral fusion revascularization is primarily derived from the surrounding muscle tissue; in addition, there is little or no compressive force on the graft material. Therefore, the distinct differences between anterior and posterior fusions must be kept in mind when critically weighing the comparative effectiveness of the different ES devices.

\section{Direct Current Electrical Stimulation}

Experimental Evidence. In 1986, Nerubay, et al., ${ }^{40}$ reported an increased rate of posterior fusion when DCES was applied in a swine model. A statistically significant increase in osteoblastic activity with bone formation was 
demonstrated in the group of animals in which ES-supplemented spinal fusion was performed. Kahanovitz and Arnoczky, ${ }^{30}$ using a canine model for posterior fusion augmented with DCES, found similar results. Fusion was assessed radiographically and histologically. At 4 and 6 weeks postoperatively, there was no significant difference between DCES-treated and control groups. At 12 weeks postoperatively, however, serial high-resolution radiographs demonstrated complete fusion in stimulated samples whereas none was observed in controls. Histological examination at 12 weeks demonstrated evidence of solid fusion in all stimulated samples and none in control specimens, a statistically significant result. The long-term DCES-related results indicated that all stimulated subjects achieved solid fusion compared with none in the control group. ${ }^{30}$

Further studies have confirmed the beneficial effects of DCES on spinal fusion. The effects of DCES on improving fusion success of coraline hydroxyapatite-assisted fusion in which a high-current direct-current stimulator is used showed improved fusion rates and mechanical stiffness compared with autograft-treated controls receiving no DCES. ${ }^{12}$ In another study, investigators examined the effects of increasing the current density delivered via DCES in the rabbit model of posterior spinal fusion mass. By increasing the current from $20 \mu \mathrm{A}$ to $60 \mu \mathrm{A}$ the healing rate and fusion mass strength increased. Higher current densities resulted in statistically significant evidence of faster fusion formation. These results were confirmed histologically, radiographically, and biomechanically. ${ }^{21}$ Caution should be exercised when increasing the current because that over $100 \mu \mathrm{A}$ is known to cause osteonecrosis and soft-tissue injury, both local conditions that could result in pseudarthrosis. The authors of a more recent controlled experimental study further expanded the potential use of ES. In sheep undergoing titanium cage-assisted anterior interbody fusion, the provision of DCES showed a statistically significant dose-dependent increase in the time to fusion, based on histological, radiographic, and biomechanical results. ${ }^{45}$ Although these attempts to expand the use of DCES are limited to a few animal studies, it is apparent that the ultimate potential, at least for DCES, has not been realized.

Clinical Evidence. In 1988, Kane ${ }^{33}$ was the first to publish a large multicenter series of patients undergoing DCES-agumented posterior fusion for a variety of spinal disorders. This publication actually contained the results of three independent clinical studies. In the first study he reported the results obtained in 82 patients undergoing DCES-assisted posterior fusion compared with historical control group of 150 patients who underwent fusion alone. Fusion was assessed radiographically at 12 and 18 months postoperatively by the operating surgeon and a radiologist. The DCES-treated group was found to have a statistically higher success rate of $91 \%$ compared with $81 \%$ in the non-DCES-treated control group, despite the fact that the former group had a significantly higher incidence of pseudarthrosis revision. In the second study the author reported a randomized prospective controlled study in a specifically defined "difficult to fuse" population consisting of: 1) one or more previous fusion attempts; 2) multilevel procedures; 3) Grade II or worse spondylolisthesis; and 4) other risk factors such as obese patients, smokers, and diabetics. Twenty-eight patients undergoing posterior fusion without stimulation were compared with 31 patients undergoing DCES-augmented fusion. In the DCEStreated group fusion rate was $81 \%$ whereas in the fusionalone group the rate was 54\% $(\mathrm{p}=0.026)$. In the third study the author examined 116 patients in an uncontrolled trial of posterior fusion augmented with DCES in the same "difficult" population. The overall fusion rate was $93 \%$.

Over the last 5 years, several additional clinical studies specifically designed to assess the efficacy of DCES on lumbar spinal fusion have been published. In 1996 Meril $^{38}$ reported the results obtained in patients who underwent anterior and posterior lumbar interbody fusion with and without DCES. Overall the fusion rates were found to be 95\% in the DCES-augmented stimulated group and $75 \%$ in the non-DCES-treated group, a statistically significant result. In DCES-treated patients the success rates were higher in all patient subgroups. Particularly interesting was the success rate among patients who were smokers (93\%) compared with that in non-DCES-treated patients (71\%).

In the remaining studies investigators have focused on the results of DCES-assisted posterolateral fusion. In one study published in 1996, the authors reported a success rate of $96 \%$ in patients undergoing posterior fusion in which pedicle screw instrumentation and adjunctive DCES were used, whereas in those who underwent pedicle screw-assisted fusion alone the rate was $85 \% .{ }^{41} \mathrm{~A}$ similar study was conducted in 1999 to examine the adjunctive use of DCES in patients undergoing pedicle screw-assisted posterior spinal fusion; the authors reported fusion rates of 95 and $87 \%$ in the DCES-treated and fusion-alone groups, respectively. In this study, smokers in the DCES-treated group fared much better than those without DCES (83 and 66\%, respectively). In cases involving fusions augmented with DCES a statistically significant increase in the clinical success and significantly higher fusion grades were reported. ${ }^{34}$ Thus, both radiographically and clinically, there appears to be significant benefit for the concomitant use of both DCES and instrumentation. A beneficial effect of DCES has also been reporting in patients undergoing noninstrumented posterior spinal arthrodesis. A 1996 prospective study was conducted in 118 patients undergoing multilevel posterior spinal DCES-augmented arthrodesis. In cases treated with and without pedicle screw instrumentation, success rates varied between 91 and $93 \%$ in a median 5-year follow-up period (range $2-10$ years). ${ }^{44}$

Direct Current Electrical Stimulation Compared with PEMF. The authors of a recent clinical study compared the use of DCES and PEMF in patients undergoing instrumentation-assisted posterior lumbar fusion. ${ }^{29}$ One year after surgery, the fusion rates were not statistically different between the groups. The only statistically significant difference was that increased BMD at their fusion site was demonstrated in both ES-treated groups compared with nonstimulated controls. The significance of increase BMD was not known. The effectiveness of DCES in enhancing spinal fusion has been demonstrated in numerous clinical reports despite our not knowing the biological mechanism by which DCES enhances spinal fusion. Further, its effectiveness has been especially demonstrated in patients in whom risk factors that make bone healing difficult. 


\section{Pulsed Electromagnetic Fields}

Experimental Evidence. Experimental evidence for the use of PEMF after spinal fusion is not as conclusive in animal models as it is with DCES. Kahanovitz, et al., ${ }^{31}$ published the first controlled experimental clinical study to demonstrate no long-term benefit in posterior spinal fusion in canines when postoperative PEMF was applied despite an encouraging but inconclusive early accelerated healing response. Specifically, they found no any statistically significant increased fusion rate between animals exposed to PEMF and those not exposed. The authors, however, did demonstrate earlier callus formation as well as remodeling of the shape of the graft in PEMF-treated animals. At later time points these differences were no longer apparent, and the intergroup fusion rates were similar. Similarly, in a histological study conducted by Guizzardi, et al., ${ }^{26}$ the author found that rats in which posterior fusion was performed demonstrated enhanced bone callus formation at 4 and 8 weeks postoperatively when compared with controls.

In 1994 Kahanovitz, et al., ${ }^{32}$ reported on additional experimental studies in which they examined if postoperative exposure to PEMF increased the fusion rate. In this second attempt by these investigators, no significant differences in the fusion rate were observed between PEMFexposed and fusion-only-treated canines. In this study, in which the investigators substituted a fresh-fracture healing PEMF for the previously used bone-healing PEMF, there was similarly no evidence of an enhanced fusion rate. ${ }^{31,32}$ A different group study examined the effect of PEMF on instrumentation-assisted posterolateral fusion in beagles. ${ }^{28}$ The authors reported a $17 \%$ change in BMD of the vertebral bodies in the animals in which instrumentation was placed but found no statistically significant improvement in BMD in relation to PEMF.

In contrast to the aforementioned studies, Glazer, et al. ${ }^{24}$ examined the use of PEMF to increase the fusion rate in a rabbit posterolateral fusion model. They were able to demonstrate the benefit of PEMF after spinal arthrodesis. They found that the fusion rate was increased in rabbits exposed to a 6-week, 4-hour/day course of PEMF after spinal fusion, based on histological, radiographic, and biomechanical evidence.

Clinical Evidence. In 1985, Simmons, et al., ${ }^{43}$ was the first to report the clinical efficacy of PEMF-augmented spinal fusion. He described the effects of PEMF on established pseudarthrosis in 13 patients who had undergone posterior lumbar interbody fusion. Without additional reparative surgery, healed interbody pseudarthrosis was found in $77 \%$ of the patients. ${ }^{43}$ Unlike the experimental studies in which the animals were exposed to PEMF in the immediate postoperative period, Simmons applied PEMF much later after surgery, when it was possible to determine if fusion had failed, a pseudarthrosis had developed, or if there was a delay in fusion.

Using "delayed" PEMF, Lee (Lee K, unpublished data) in 1989 reported in an abstract the results of patients treated for posterior pseudarthrosis with adjunctive PEMF. He reported a fusion rate of $67 \%$, which was not as high as the $77 \%$ rate noted by Simmons, et al. ${ }^{43}$ in patients treated for anterior interbody pseudarthrosis. The fusion rate depend- ed on the compliance of the patients in wearing the PEMF device. In noncompliant patients the fusion rates were similar to non-PEMF-treated controls, whereas in the most compliant patients higher fusion rates were evident. In the same year, Simmons, et al., published an abstract in which they reported on the first use of PEMF as an adjunct to primary posterolateral spinal fusion (Simmons JW, et al., unpublished data). Their patients were exposed to PEMF in the immediate postoperative period. The fusion rate of $71 \%$ was significantly lower than that demonstrated in patients undergoing primary posterolateral fusion in a different series in which DCES was used.

In 1990, Mooney ${ }^{39}$ reported on the first large multicenter series of patients treated with adjunctive PEMF. Unlike the multicenter studies of DCES-enhanced posterior spinal fusion published by Kane, ${ }^{33}$ Mooney described the results of 195 patients who underwent primary posterior or anterior lumbar interbody fusions. None of the patients underwent posterolateral spinal fusion. Overall the fusion rate of $92 \%$ was similar to Kane's overall results, but the radiographic criteria for fusion required only $50 \%$ incorporation of the graft. Further follow-up evaluation demonstrated that the success rate had decreased by approximately $24 \%$, for a fusion rate of $68 \%$.

Additional studies on the use of PEMF-assisted posterolateral spinal fusion have been reported. Savini, et al., ${ }^{42}$ studied an uncontrolled group of only 15 patients; in another study examining a controlled group of 35 patients by Mammi, et al., (Mammi GI, et al., unpublished data) demonstrated encouraging preliminary results in a followup period of less than 1 year. Longer follow-up evaluations of these patients have not been reported.

Longer-term follow-up data were reported by Marks ${ }^{37}$ in 61 patients with discogenic low-back pain who underwent fusion with or without PEMF. In the 42 patients in whom PEMF was applied, the author demonstrated a radiographic fusion rate of $97.6 \%$ within a mean follow-up period of 15 months, whereas in patients treated without PEMF the mean fusion rate was $52.6 \%$. The observed agreement between clinical and radiographic outcome was $75 \%$. Mark found that PEMF enhanced osseous bridging in lumbar spinal fusions.

In an uncontrolled study of 48 high-risk fusion patients reported in 2001 Bose $^{11}$ demonstrated that PEMF applied postoperatively resulted in a $97.9 \%$ radiographic fusion rate as determined by an independent examiner. In this study overall clinical assessment was excellent to good in $83.4 \%$ of patients.

Combined Magnetic Fields Device. In the latest clinical effort to examine the use of adjunctive CMFs to enhance noninstrumented posterolateral spinal fusion, the findings were reported at the American Academy of Orthopedic Surgeons 2000 meeting (Linovitz RJ, unpublished data). A CMF device differs from a PEMF device by superimposing the time-varying magnetic field onto an additional static magnetic field. Linovitz, et al., reported an overall success rate of $64 \%$ in the stimulated group compared with $43 \%$ in the control group. Combined magnetic fields appeared to be effective only in women; no improvement in fusion rates was observed in men. There are no other published scientific studies in which the clinical efficacy of $\mathrm{CMF}$ is documented. 


\section{Capacitive Coupling}

Clinical Evidence. The concept of using capacitively coupled ES as an adjunct to lumbar fusion is relatively new. Although it has been commercially available since the early 1990s for long bone fracture nonunions, its efficacy in spinal fusion was only recently demonstrated in a multicenter randomized double-blind study by Goodwin, et al. ${ }^{25}$ The overall fusion rate of the ES-treated patients (84\%) compared with the non-ES-treated patients $(64 \%)$ was statistically significant. Of the groups used to stratify the data, four of the seven showed statistical significance between the actively stimulated patients and the placebotreated patients. To date no other studies, including experimental studies, have been published on the effects of capacitive coupling as an adjunct to spinal fusion.

\section{CONCLUSIONS}

Over the last 30 years, ES has become clearly distinguished as a clinically beneficial adjunct to spinal fusion. It appears that ES may be most beneficial in selected patients are at greatest risk of nonunion. Many of the devices are costly (\$2500-\$7000). Because of the cost, selective use of the devices is warranted, especially those for which the experimental and clinical evidence is scant. Cases in which the spine surgeon may want to consider adjunctive ES include patients undergoing multilevel fusion or reoperation, those who use tobacco, and those with disorders of low BMD (that is, osteoporosis). Not all adjunctive ES devices, however, are equally effective in promoting fusion. In this review, an attempt was made to discuss the different devices and to review the literature on the clinical effectiveness of these devices. Both the current clinical and basic science data establish DCES as superior to PEMF in promoting bone union, particularly when used to enhance posterior spinal fusion. Indeed, in the vast majority of the reports the authors discuss lumbar spine surgery. To date, the effectiveness of ES on cervical and thoracic regions has not been reported. A newer ES modality, capacitive coupling, has only been examined in one study so far; however, its promise was shown as an adjunct to spinal fusion. In summary, DCES appears to be associated with the greatest basic science and clinical evidence indicating that it increases the rate of spinal fusion compared with the other ES modalities. The clinical effectiveness of PEMF devices in the treatment of delayed unions and pseudarthrosis has clearly been demonstrated without the need for reoperation. Although evidence regarding the efficacy of capacitive coupling devices is still lacking, it is hoped that further peer-reviewed studies will determine its effectiveness or lack thereof.

\section{References}

1. Aaron RK, Ciombor DM: Electrical stimulation of bone induction and grafting, in Habal MB, Reddi AH (eds): Bone Grafts \& Bone Substitutes. Philadelphia: WB Saunders, 1992, pp 192-205

2. Aaron RK, Ciombor DM, Jolly G: Stimulation of experimental endochondral ossification by low-energy pulsing electromagnetic fields. J Bone Miner Res 4:227-233, 1989

3. Baranowski TJ Jr, Black J: The mechanism of faradic stimulation of osteogenesis, in Blank M, Findl E (eds): Mechanistic
Approaches to Interactions of Electric and Electromagnetic Fields with Living Systems. New York: Plenum Press, 1987 pp 399-416

4. Bassett CA: Pulsing electromagnetic fields: a new method to modify cell behavior in calcified and noncalcified tissues. Calcif Tissue Int 34:1-8, 1982

5. Bassett CA, Mitchell SN, Gaston SR: Pulsing electromagnetic field treatment in ununited fractures and failed arthrodeses. JAMA 247:623-628, 1982

6. Bassett CA, Pawluk RJ, Pilla AA: Augmentation of bone repair by inductively coupled electromagnetic fields. Science 184: 575-577, 1974

7. Bassett CA, Valdes MG, Hernandez E: Modification of fracture repair with selected pulsing electromagnatic fields. J Bone Joint Surg (Am) 64:888-895, 1982

8. Bassett CAL: The role of pulsed electromgnetic fields in bone grafting, in Habal MB, Reddi AH (eds): Bone Grafts \& Bone Substitutes. Philadelphia: Saunders, 1992, pp 173-191

9. Becker RO: The significance of electrically stimulated osteogenesis: more questions than answers. Clin Orthop 141: 266-274, 1979

10. Bodamyali T, Bhatt B, Hughes FJ, et al: Pulsed electromagnetic fields simultaneously induce osteogenesis and upregulate transcription of bone morphogenetic proteins 2 and 4 in rat osteoblasts in vitro. Biochem Biophys Res Commun 250: 458-461, 1998

11. Bose B: Outcomes after posterolateral lumbar fusion with instrumentation in patients treated with adjunctive pulsed electromagnetic field stimulation. Adv Ther 18:12-20, 2001

12. Bozic KJ, Glazer PA, Zurakowski D, et al: In vivo evaluation of coralline hydroxyapatite and direct current electrical stimulation in lumbar spinal fusion. Spine 20:2127-2133, 1999

13. Brighton CT: The treatment of non-unions with electricity. $\mathbf{J}$ Bone Joint Surg (Am) 63:847-851, 1981

14. Brighton CT, Friedenberg ZB: Electrical stimulation and oxygen tension. Ann NY Acad Sci 238:314-320, 1974

15. Brighton CT, Friedenberg ZB, Black J, et al: Electrically induced osteogenesis: relationship between charge, current density, and the amount of bone formed: introduction of a new cathode concept. Clin Orthop 161:122-132, 1981

16. Brighton CT, Heppenstall RB: Oxygen tension in zones of the epiphyseal plate, the metaphysis and diaphysis. An in vitro and in vivo study in rats and rabbits. J Bone Joint Surg (Am) 53: 719-728, 1971

17. Cleveland M, Bosworth DM, Thompson FR: Pseudarthrosis in the lumbosacral spine. J Bone Joint Surg (Am) 30:302-312, 1948

18. Dwyer AF, Yau AC, Jeffcoat KW: Use of direct current in spine fusion. J Bone Joint Surg (Am) 56A:442, 1974 (Abstract)

19. Ferrier J, Ross SM, Kanehisa J, et al: Osteoclasts and osteoblasts migrate in opposite directions in response to a constant electrical field. J Cell Physiol 129:283-288, 1986

20. Fitzsimmons RJ, Strong DD, Mohan S, et al: Low-amplitude, low-frequency electric field-stimulated bone cell proliferation my in part be mediated by increased IGF-II release. J Cell Physiol 150:84-89, 1992

21. France JC, Norman TL, Santrock RD, et al: The efficacy of direct current stimulation for lumbar intertransverse process fusions in an animal model. Spine 26:1002-1008, 2001

22. Friedenberg ZB, Brighton CT: Bioelectric potentials in bone. $\mathbf{J}$ Bone Joint Surg (Am) 48:915-923, 1966

23. Fukuda E, Yasuda I: On the piezoelectric effect of bone. J Physiol Soc Jpn 12:1158-1162, 1957 (reference unverified)

24. Glazer PA, Heilmann MR, Lotz JC, et al: Use of electromagnetic fields in spinal fusion. A rabbit model. Spine 22: 2351-2356, 1997

25. Goodwin CB, Brighton CT, Guyer RD, et al: A double-blind study of capacitively coupled electrical stimulation as an adjunct to lumbar spinal fusions. Spine 24:1349-1357, 1999 
26. Guizzardi S, Di Silvestre M, Govoni P, et al: Pulsed electromagnetic field stimulation on posterior spinal fusions: a histological study in rats. J Spinal Disord 7:36-40, 1994

27. Hastings GW, Mahmud FA: Electrical effects in bone. J Biomed Eng 10:515-521, 1988

28. Ito M, Fay LA, Ito Y, et al: The effect of pulsed electromagnetic fields on instrumented posterolateral spinal fusion and device-related stress shielding. Spine 22:382-388, 1997

29. Jenis LG, An HS, Stein R, et al: Prospective comparison of the effect of direct current electrical stimulation and pulsed electromagnetic fields on instrumented posterolateral lumbar arthrodesis. J Spinal Disord 13:290-296, 2000

30. Kahanovitz N, Arnoczky SP: The efficacy of direct current electrical stimulation to enhance canine spinal fusions. Clin Orthop 251:295-299, 1990

31. Kahanovitz N, Arnoczky SP, Hulse D, et al: The effect of postoperative electromagnetic pulsing on canine posterior spinal fusions. Spine 9:273-279, 1984

32. Kahanovitz N, Arnoczky SP, Nemzek J, et al: The effect of electromagnetic pulsing on posterior lumbar spinal fusions in dogs. Spine 19:705-709, 1994

33. Kane WJ: Direct current electrical bone growth stimulation for spinal fusion. Spine 13:363-365, 1988

34. Kucharzyk DW: A controlled prospective outcome study of implantable electrical stimulation with spinal instrumentation in a high-risk spinal fusion population. Spine 24:465-469, 1999

35. Lohmann CH, Schwartz Z, Liu Y, et al: Pulsed electromagnetic field stimulation of MG63 osteoblast-like cells affects differentiation and local factor production. J Orthop Res 18: 637-646, 2000

36. Lorich DG, Brighton CT, Gupta R, et al: Biochemical pathway mediating the response of bone cells to capacitive coupling. Clin Orthop 350:246-256, 1998

37. Marks RA: Spine fusion for discogenic low back pain: outcomes in patients treated with or without pulsed electromagnetic field stimulation. Adv Ther 17:57-67, 2000

38. Meril AJ: Direct current (DC) stimulation of allograft in anterior and posterior lumbar interbody fusions. Spine 19: 2393-2398, 1994
39. Mooney V: A randomized double-blind prospective study of the efficacy of pulsed electromagnetic fields for interbody lumbar fusions. Spine 15:708-712, 1990

40. Nerubay J, Marganit B, Bubis JJ, et al: Stimulation of bone formation by electircal current on spial fusion. Spine 11:167-169, 1986

41. Rogozinski A, Rogozinski C: Efficacy of implanted bone growth stimulation in instrumented lumbosacral spinal fusion. Spine 21:2479-2483, 1996

42. Savini R, DiSilvestre M, Gargiulo G, et al: The use of pulsing electromagnetic fields in posterolateral lumbosacral spinal fusion. J Bioelectricity 9:9-17, 1990 (reference unverified)

43. Simmons JW: Treatment of failed posterior lumbar interbody fusion (PLIF) of the spine with pulsing electromagnetic fields. Clin Orthop 193:127-132, 1985

44. Tejano NA, Puno R, Ignacio JM: The use of implantable direct current stimulation in multilevel spinal fusion without instrumentation. A prospective clinical and radiographic evaluation with long-term follow-up. Spine 21:1904-1908, 1996

45. Toth JM, Seim HB III, Schwardt JD, et al: Direct current electrical stimulation increases the fusion rate of spinal fusion cages. Spine 25:2580-2587, 2000

46. Yasuda I: On the piezoelectric property of bone. J Jpn Orthop Surg Soc 28:267-279, 1954

47. Yen-Patton GP, Patton WF, Beer DM, et al: Endothelial cell response to pulsed electromagnetic fields: stimulation of growth rate and angiogenesis in vitro. J Cell Physiol 134:37-46, 1988

48. Zhuang H, Wang W, Seldes RM, et al: Electrical stimulation induces the level of TGF-beta1 mRNA in osteoblastic cells by a mechanism involving calcium/calmodulin pathway. Biochem Biophys Res Commun 237:225-229, 1997

Manuscript received October 10, 2002.

Accepted in final form November 20, 2002.

Address reprint requests to: Michael A. Morone, M.D., Ph.D., Deaconess Billings Clinic, 2825 8th Avenue North, PO Box 37000, Billings, MT 59107-7000. email: mmorone@billingsclinic.org. 